UCRL-ID-123083

\title{
SSD with Generalized Phase Modulation
}

J. Rothenberg

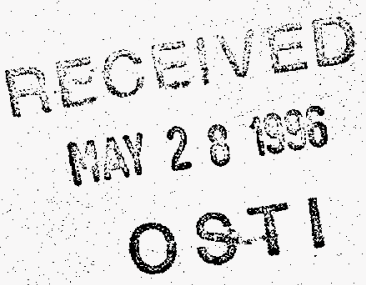

January 9, 1996

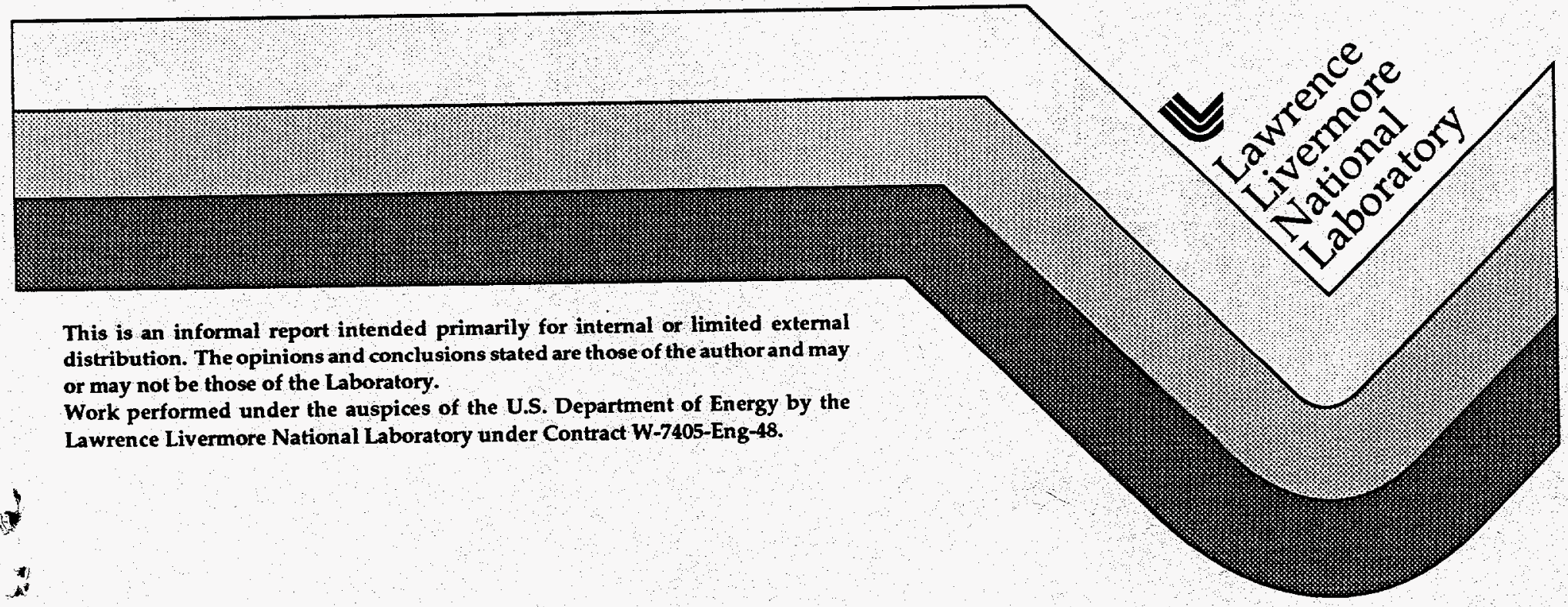




\section{DISCLAIMER}

This document was prepared as an account of work sponsored by an agency of the United States Government. Neither the United States Government nor the University of California nor any of their employees, makes any warranty, express or implied, or assumes any legal liability or responsibility for the accuracy, completeness, or usefulness of any information, apparatus, product, or process disclosed, or represents that its use would not infringe privately owned rights. Reference herein to any specific commercial product, process, or service by trade name, trademark, manufacturer, or otherwise, does not necessarily constitute or imply its endorsement, recommendation, or favoring by the United States Government or the University of California. The views and opinions of authors expressed herein do not necessarily state or reflect those of the United States Government or the University of California, and shall not be used for advertising or product endorsement purposes.

This report has been reproduced directly from the best available copy.

Available to DOE and DOE contractors from the

Office of Scientific and Technical Information

P.O. Box 62, Oak Ridge, TN 37831

Prices available from (615) 576-8401, FTS 626-8401

Available to the public from the

National Technical Information Service

U.S. Department of Commerce

5285 Port Royal Rd.

Springfield, VA 22161 
Interdepartmental letterhead

Mail Station L

493

Ext: $3-8613$

NIF-LLNL-96-002

L-21145-01

December 27, 1995

TO: $\quad$ Distribution

FROM: Josh Rothenberg 8

SUBJECT: SSD with generalized phase modulation

Summary: Smoothing by spectral dispersion (SSD) with standard frequency modulation (FM), although most simple to implement, has the significant disadvantage that low spatial frequencies present in the spectrum of the target illumination are not smoothed as effectively as is possible with a more general smoothing method (such as the induced spatial incoherence (ISI) method). The reduced smoothing performance of standard FM-SSD can result in spectral power of the speckle noise at these low spatial frequencies as much as one order of magnitude larger than that achieved with a more general method. In fact, at small integration times FM-SSD has no smoothing effect at all for a broad band of low spatial frequencies. This effect may have important implications for both direct and indirect drive ICF.

This disadvantage of standard FM-SSD can be overcome by using more general phase modulation. With this approach one can achieve optimal smoothing at low spatial frequencies and also maintain the near field beam quality, since one still utilizes pure phase modulation. The essential ingredient necessary in order to achieve optimal smoothing at low spatial frequency is the generation of many "color cycles" across the beam. That is, the temporal skew imposed by the SSD grating must be many times larger than the mean period of the phase modulation. If, however, one simply uses sinusoidal FM of many color cycles, coherence resonances appear in the spatial spectrum of the target illumination owing to the pure periodicity of the FM. By generating many color cycles and also "randomizing" the phase modulation one can eliminate these resonances and still obtain optimum smoothing at low spatial frequency. The spatial spectrum of the smoothed illumination of SSD using random phase modulation (RPM) is equivalent to that of an ISI method of equal divergence. Instead of RPM, a potentially simpler and more practical approach to eliminating these resonances is the use of additional FM to spoil the pure periodicity of a single FM.
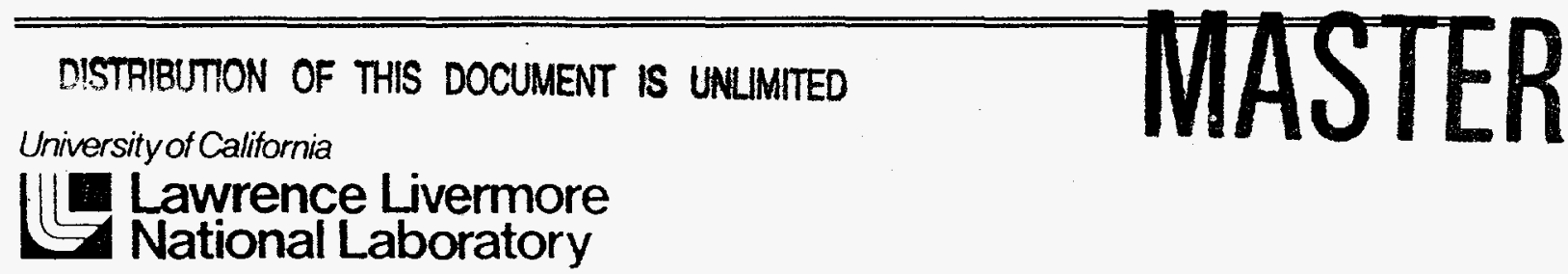
The technique of SSD utilizing generalized phase modulation is depicted in Fig. 1. The beam is modulated with some phase function $\phi(t)$ and then dispersed by a grating before amplification. The effect of the dispersion is to time skew the phase function across the beam. I.e. the phase after dispersion is described by $\phi(t-s x)$, where the time skew per unit distance $s$ is proportional to the grating dispersion. For 2D SSD a second modulator and orthogonal grating are added. A random phase plate (RPP) is used to determine the spot profile on the target. The great advantage of the SSD method is that in and near the relay image planes of the grating the pure phase modulation imposed does not lead to amplitude modulation and thus high power amplification is made more efficient.

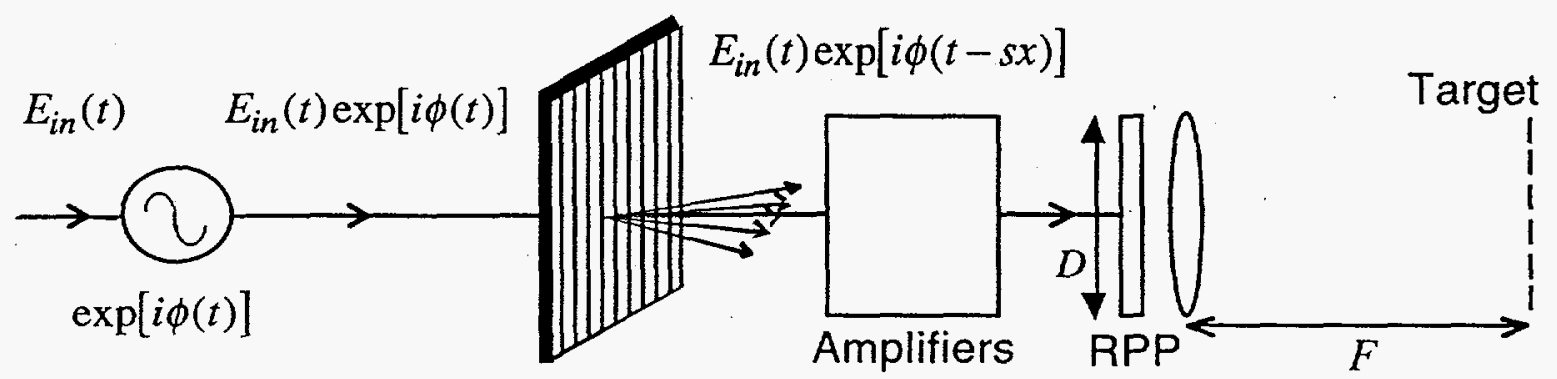

Figure 1: Schematic layout of 1D-SSD with generalized phase modulation.

For a completely randomized smoothing method (e.g. ISI with a very large beam divergence), where the speckle patterns at successive coherence times are completely uncorrelated, one ideally finds that all spatial frequencies are smoothed at the same bandwidth limited rate. The rate is determined by the number of independent speckle patterns which are generated in an integration time $t$. Assuming Gaussian statistics, one can show that for a smoothing method with spectral intensity $I(v)$, the effective smoothing rate is generally given by

$$
\Delta v_{\text {eff }}=\left|\int I(v) d v\right|^{2} / \int|I(v)|^{2} d v \text {. }
$$

For a spectrum of Gaussian shape and FWHM $\Delta v_{f w}$, one finds that $\Delta v_{e f f}=1.5 \Delta v_{f w}$, the coherence time is $\sim 1 /\left(1.5 \Delta v_{f w}\right)$, and hence the number of speckle patterns generated in an integration time $t$ is $\sim 1.5 \Delta v_{f w} t$. Thus, in this case, the spectral intensity of the speckle noise at any spatial frequency will be reduced by this factor. However, for any realistic smoothing method the beam divergence is limited. Hence, the extent of the speckle motion is limited and therefore there will always be a low spatial frequency limit below which no smoothing occurs (this limit is present regardless of the smoothing method used).

\section{Smoothing by the random ISI method}

This behavior is illustrated in Fig. 2, where the spatial power spectra of the integrated intensity on target vs. normalized spatial frequency are shown at a series of integration times for a random smoothing method (ISI) with a beam 
divergence of Gaussian FWHM 50 / D. The dashed curves show the ideal behavior of random smoothing of unlimited beam divergence, and are simply the initial static speckle spectrum divided by the number of coherence times (i.e. $1.5 \Delta v t)$. One sees that at large spatial frequency the ideal smoothing limit is achieved. However, below a spatial frequency determined by the induced divergence there is little or no smoothing.

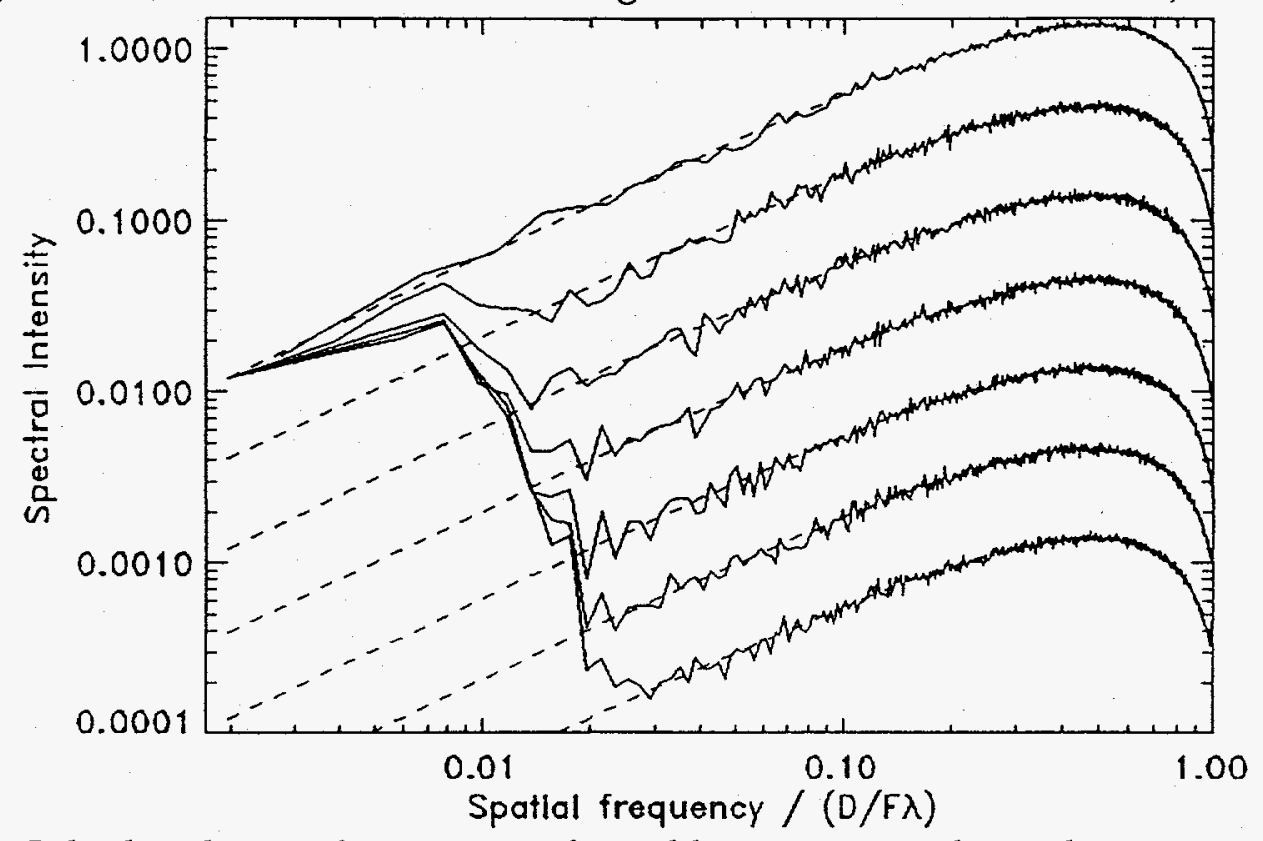

Fig. 2: Calculated spatial spectrum of speckle patterns with random smoothing (ISI) of divergence FWHM $50 \lambda / D$ for (top to bottom curves) the initial static speckle pattern and after an integration time equivalent to $3,10,30,100,300$, and 1000 coherence times. The dashed lines show the ideal smoothing result for the case of large beam divergence.

In this calculation the speckle patterns at successive coherence times are assumed completely uncorellated, except that they are limited within a given beam divergence. In all the calculations presented here an RPP or amplifier phase aberrations are assumed to produce a far field spot larger than the wavelengths considered. The spatial frequency scale shown is normalized to the F\# limited spatial frequency $f_{0} \equiv D / \lambda F$, where $D$ is the final near field aperture width, $F$ is the final lens focal length, and $\lambda$ is the wavelength on target (see Fig. 1). For the NIF focusing geometry $(D=35 \mathrm{~cm}, F=7 \mathrm{~m}) \lambda / D=1 \mu \mathrm{rad}, f_{0}$ corresponds to a wavelength of $7 \mu \mathrm{m}$, and for a direct drive target of radius $1.6 \mathrm{~mm} f_{0}$ corresponds to an $\ell$-mode of 1500 . These spectra have been integrated over azimuth in the spectral plane $\left(f_{x}, f_{y}\right)$ so that they are only dependent on the magnitude of the spatial frequency (or $\ell$-mode), and are normalized such that the integral over the normalized frequency yields the normalized variance ${ }^{1}\left(\sigma^{2}\right)$ which is unity for a static speckle pattern. 


\section{D SSD using standard FM}

The spatial spectra generated by 2D-SSD using standard FM are significantly different. For comparison, in Fig. 3 the spatial spectra produced by 2D-SSD using FM with a single color cycle in each dispersion direction (i.e. where the temporal skew of the grating is equal to the modulator period; dotted curves) are shown with the results of the ISI random smoothing of Fig. 2 (solid curves), where both smoothing methods are chosen to generate the same beam divergence FWHM (note that the angular spectrum of the FM-SSD beam has a rectangular shape peaked at the ends (characteristic of a FM spectrum) whereas the ISI random smoothing described here has a Gaussian angular spectrum). One sees that FM-SSD (unless otherwise specified SSD refers to 2D SSD) smoothes comparatively poorly at low spatial frequency. At each integration time the FM-SSD spectral intensity is seen to be slightly smaller than that of the ISI random smoothing at high spatial frequency, but crosses over near $0.25 f_{0}$. At spatial frequencies $\sim 0.02 f_{0}-0.06 f_{0}$ the FM-SSD spectral intensity is 5-10 times larger than that produced by the random ISI method.

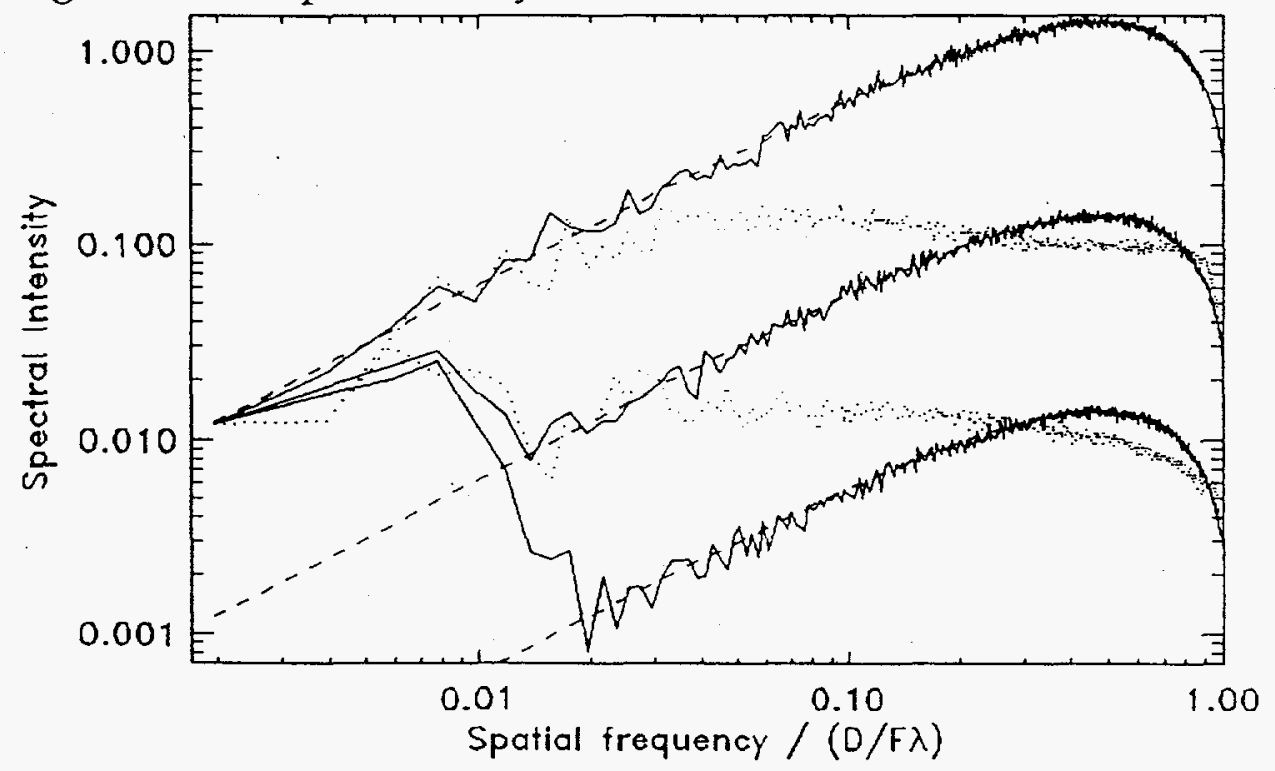

Fig. 3: (a) Comparison of calculated spatial spectra using smoothing by ISI of divergence FWHM $50 \lambda / D$ (solid curves) with that of standard FM-2D SSD of equal divergence (dotted curves) for (top to bottom) the initial static speckle pattern (solid) and after an integration time equivalent to 10 and 100 coherence times. The dashed lines show the ideal random smoothing result for the case of large beam divergence.

\section{SSD with Random Phase Modulation}

As seen in Fig. 3, single color cycle FM-SSD, because of its coherence properties, has much lower smoothing rates at low spatial frequencies than the ISI method of equal beam divergence. One can show that the smoothing rate of SSD at low spatial frequency can be improved by increasing the number of color cycles 
across the beam. The number of color cycles across the beam $N_{c c}$ is defined as the number of FM periods ( $T_{\text {mod }}$ ) in the total time skew provided by the grating $\left(T_{\text {skew }}\right)$

$$
N_{c c}=T_{\text {skew }} / T_{\text {mod }}=T_{\text {skew }} \cdot v_{\text {mod }},
$$

where $v_{\text {mod }}$ is the modulation frequency. The smoothing rate dependence on $N_{c c}$ is understood if one realizes that the low spatial frequencies on the target originate from closely separated points in the near field (points separated by $\Delta x$ in the near field contribute to spatial frequency on the target given by $f=\Delta x(F \lambda)$. As $N_{c c}$ is increased, the relative coherence of closely separated near field points decreases, and thus one obtains a larger smoothing rate at low spatial frequency. However, by imposing multiple color cycles with pure sinusoidal phase modulation one creates multiple bands across the beam which are coherent with each other. The coherent interference between these bands leads to zero or reduced smoothing at the spatial frequencies corresponding to the band separations. By randomizing the phase modulation such that, on average, there are still many color cycles across the beam, rapid smoothing at low spatial frequency can be obtained without the presence of coherence resonances in the spatial spectrum. A more detailed analysis of multiple color cycles is given in the next section.

Figure 4 shows the spatial spectra of 2D-SSD using random phase modulation (RPM), where the RPM is simulated by filtering the white noise spectrum of a random binary sequence with a Gaussian shaped filter. In this calculation the FWHM of the phase spectrum is taken to be $50 \mathrm{Ghz}$ and the phase depth is chosen to have an RMS of $3.75 \mathrm{rad}$, which results in a total bandwidth of $185 \mathrm{Ghz}$ for each direction of the 2D-SSD. The grating skew is taken to be 280 ps so that the number of color cycles is $\sim 14$ (since the mean period of the phase modulation is $\sim 1 / 50 \mathrm{Ghz}=20 \mathrm{psec}$ ) and the beam has a Gaussian angular spread of FWHM $50 \lambda / D$. The spatial spectra produced by SSD using RPM (solid curves) is compared in Fig. 4 with SSD using FM of a single color cycle (dotted curves), where the beam divergence FWHM of the two methods is equal. One sees that the RPM-SSD method generates essentially the same spatial spectra as that of the random ISI method of equal divergence (e.g., compare with Fig. 3), and that, over spatial frequencies $0.02 f_{0}-0.06 f_{0}$, the spectral intensity produced by RPM-SSD is smaller than that of FM-SSD by a factor of $\sim 5-10$. This band of low spatial frequencies ( $\ell$-modes of 30-100) is particularly important with regard to direct drive hydrodynamic stability. Also, as shown in more detail in Fig 4(b), at very short integration times FM-SSD does not smooth a broad range of low spatial frequencies of the static speckle spectrum at all, whereas the RPM-SSD method smoothes low spatial frequencies uniformly to the limit imposed by beam divergence. This feature may be particularly important to the suppression of laser-plasma instabilities, where the smoothing rate during the first few tens of ps is most critical. From this figure one can see that the low spatial frequency 
limit where smoothing is first noticeable is decreasing linearly with integration time. It will be shown that smoothing is significant only for spatial frequencies

$$
f>f_{0} / 2 \pi N_{c c} T_{c t},
$$

where $T_{c t}$ is the integration time given as the equivalent number of coherence times.
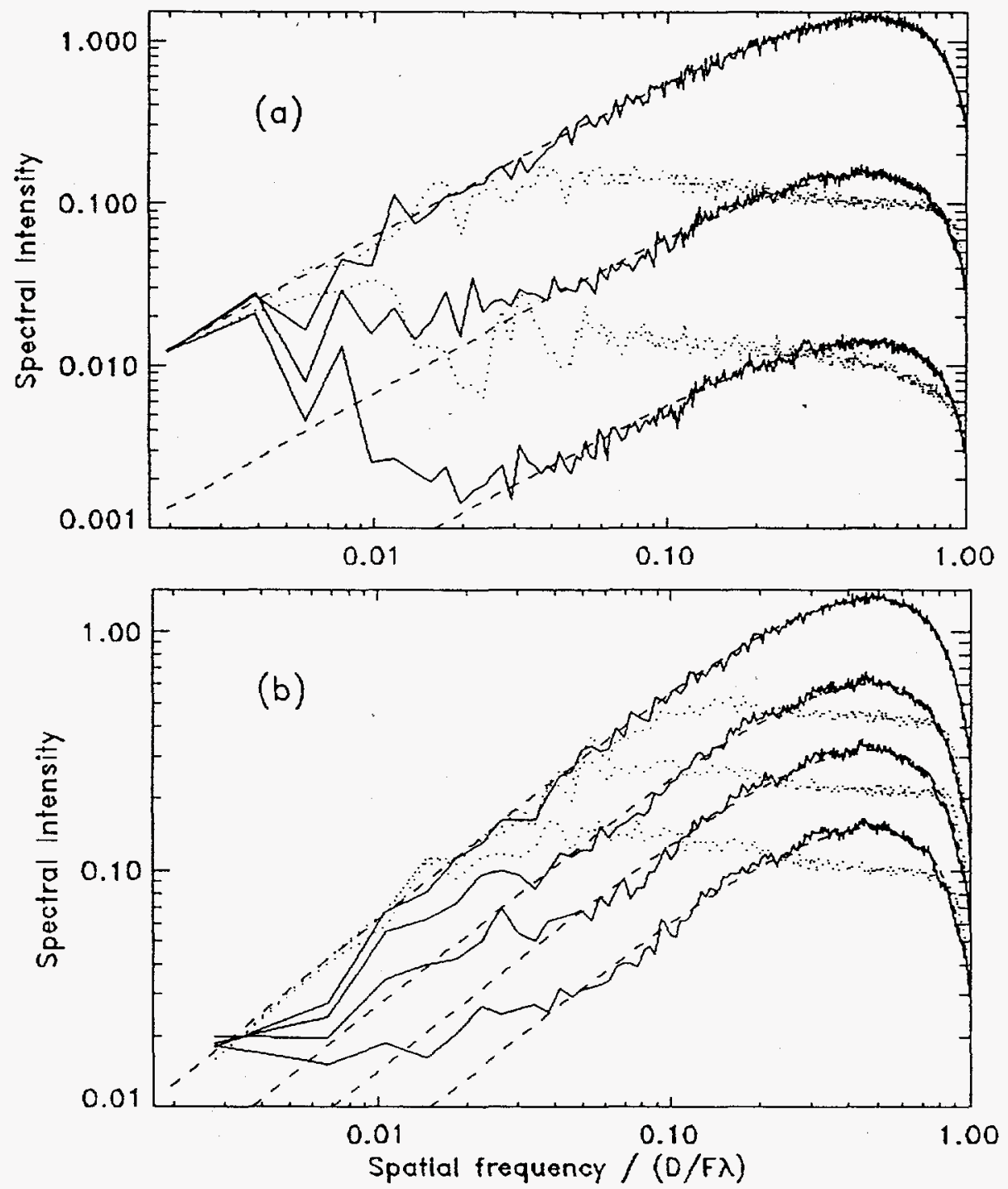

Figure 4: Comparison of calculated spatial spectra using 2D SSD with random phase modulation of $\sim 14$ color cycles across the beam (solid curves) and single color cycle FM (dotted curves) of equal beam divergence for (top to bottom) the initial static speckle pattern (solid) and after an integration time equivalent to (a) 10 and 100, and (b) 2,4, and 9 coherence times. The dashed curves show the ideal random smoothing result using large beam divergence.

As noted above, the smoothing rate of SSD at low spatial frequencies is critically dependent on the number of color cycles across the beam. Since RPM can not be described by a single frequency modulation, the length of a color cycle must be given by the mean phase oscillation period or the phase correlation length across 
the beam. One can show that the mean number of color cycles across the beam is given by the bandwidth of the phase multiplied by the temporal skew across the beam, $N_{c c}=\Delta v_{\text {phase }} \cdot T_{\text {skew }}$. Fig. 5 compares the results of smoothing using RPM for three different values of the mean number of color cycles $(2.7,6.8$, and 14), and where the bandwidth and divergence are kept constant. One can see that as the number of color cycles is increased the smoothing obtained at low spatial frequency is more rapid. In the limit of a single color cycle, RPM gives results which are similar to that obtained with FM of a single color cycle.

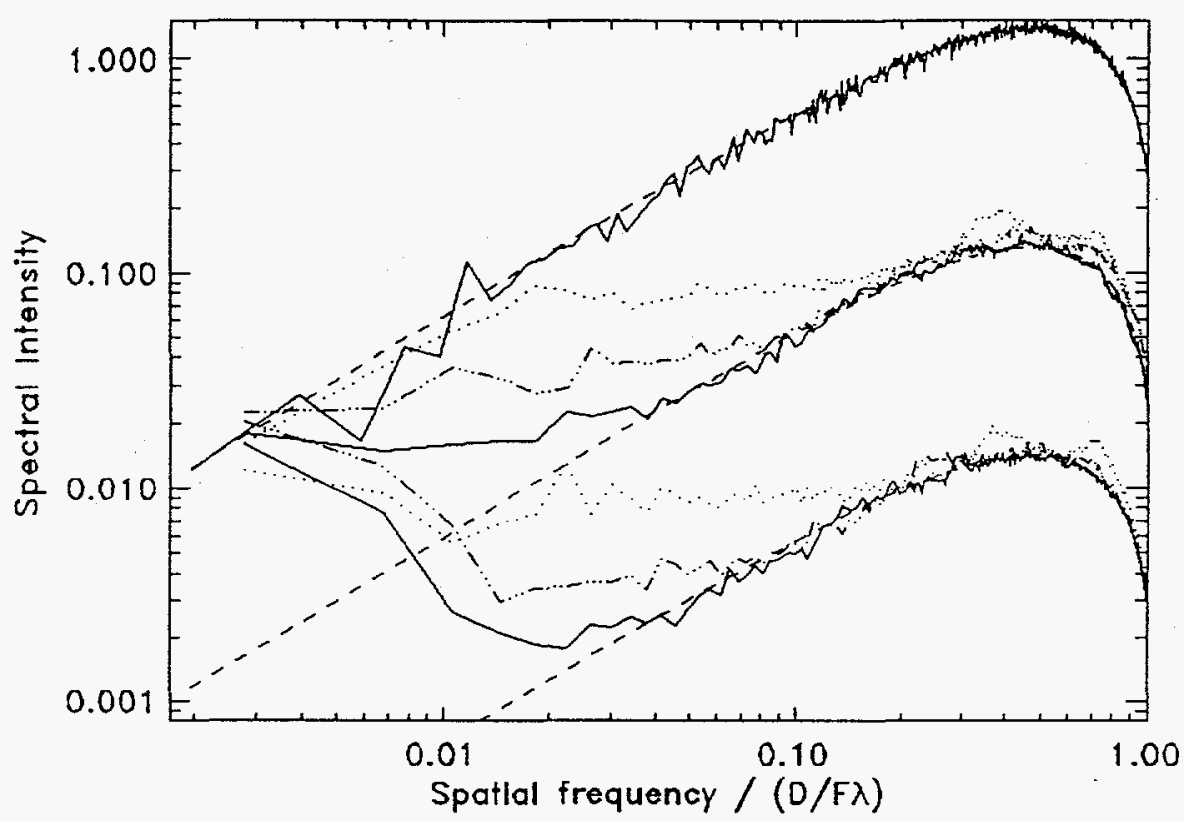

Fig. 5: SSD with random phase modulation of 14 (solid curves), 6.8 (dash-dot curves) and 2.7 (dotted curves) color cycles, for (top to bottom) the initial static speckle pattern (solid) and after an integration time equivalent to 10 and 100 coherence times. The beam divergence in each calculation is $50 \lambda / D$. The dashed curves show the ideal random smoothing result using large beam divergence.

\section{Smoothing rate dependence on color cycling}

To understand the smoothing rate dependence on the number of color cycles consider sinusoidal FM with $N_{c c}$ color cycles across the beam. The near field can be written

$$
E(x)=\exp \left\{i \beta \sin \left(k_{x} x-\omega_{\bmod } t\right)\right\}
$$

where, $k_{x}=2 \pi N_{c c} / D, \omega_{\bmod }=2 \pi v_{\text {mod }}$ is the angular modulation frequency, and the $y$ dependence has been omitted for simplicity but is analogous. Now, points separated by $\Delta x$ in the near field plane of the focusing lens contribute to spatial frequency on the target given by

$$
f=\Delta x / F \lambda
$$


Thus, one can find the smoothing rate at a given spatial frequency $f$ by calculating the interference between the field at points of the corresponding separation $\Delta x$. The intensity owing to interference of near field points separated by $\Delta x$ is given by

$$
\begin{aligned}
E(x) E^{*}(x+\Delta x) & =\exp \left\{i \beta\left[\sin \left(k_{x} x-\omega_{\bmod } t\right)-\sin \left(k_{x}(x+\Delta x)-\omega_{\bmod } t\right)\right]\right\} \\
& =\exp \left\{i 2 \beta \sin \left(k_{x} \Delta x / 2\right) \cos \left(k_{x}(x+\Delta x / 2)-\omega_{\bmod } t\right)\right\}
\end{aligned}
$$

Low spatial frequencies on the target correspond to small $\Delta x$, so that one can approximate $\sin \left(k_{x} \Delta x / 2\right) \cong k_{x} \Delta x / 2$. Hence, Eq. (5) is simplified to

$$
E(x) E^{*}(x+\Delta x)=\exp \left\{i\left(\beta k_{x} \Delta x\right) \cos \left(k_{x}(x+\Delta x / 2)-\omega_{\bmod } t\right)\right\}
$$

The interference term is recognized as having the same form as FM with the effective depth given by $\beta_{\text {eff }}(f)=\beta k_{x} \Delta x$. The bandwidth of this interference term gives the effective rate in smoothing the spatial frequency of interest (a more precise analysis involves calculating the time average of Eq. (6) and summing over the relevant aperture positions, however the heuristic approach presented here gives an accurate estimate of the smoothing rate). Thus, the effective smoothing rate can be approximated by

$$
\Delta v_{\text {eff }}(f) \sim 2 \beta_{\text {eff }}(f) \cdot v_{\text {mod }}=2 \beta v_{\text {mod }} \cdot 2 \pi N_{c c} \Delta x / D=\Delta v_{\text {tot }} \cdot 2 \pi N_{c c} \cdot \Delta x / D .
$$

where the total bandwidth is $\Delta v_{\text {tot }} \equiv 2 \beta v_{\text {mod }}$. Therefore, smoothing at spatial frequency $f=\Delta x / F \lambda$ will become significant when the integration time $t$ is sufficient such that average over the effective bandwidth starts to wash out, or when $\Delta v_{\text {eff }}(f) \cdot t \sim 1$. Hence, at integration time $t$, smoothing is significant for spatial frequencies

$$
f>(D / \lambda F) / 2 \pi N_{c c} \Delta v_{t o t} t=f_{0} / 2 \pi N_{c c} \Delta v_{t o t} t=f_{0} / 2 \pi N_{c c} T_{c t},
$$

where $T_{c t}=\Delta v_{t o t} t$ is the number of coherence times. This estimate is confirmed in Figs. 4 and 5. For example, in Fig. 4 with FM of a single color cycle, one has that at 2 coherence times the smoothing is significant only for $f>f_{0} / 4 \pi$.

The behavior of 1D-SSD with multiple color cycles is shown in Fig. 6(a), where the spatial spectrum of smoothing after $\sim 4$ coherence times using FM with one color cycle is compared with that of FM using 4 color cycles. For clarity, these spectra have not been integrated azimuthally, but are integrated orthogonally to the dispersion direction and plotted versus the spatial frequency along the dispersion direction. One sees that the smoothing at low spatial frequency is more effective when using four color cycles as predicted by Eqs. (7) and (8). However, the effect of the coherence between the periodic color cycles is also readily apparent. From Eq. (5) one sees that these resonances occur when $\sin \left(k_{x} \Delta x / 2\right)=0$, i.e. $k_{x} \Delta x=2 m \pi$ or $\Delta x=D \cdot m / N_{c c}$ (where $m$ is an integer). The coherent interference at these separations results in zero smoothing at the corresponding spatial frequencies given by $f_{0} \cdot m / N_{c c}$ (i.e. for $N_{c c}=4$, at spatial frequencies $.25, .5$, and $.75 f_{0}$ ). 
Since this effect is a result of the particular periodic coherence of pure sinusoidal FM, the superposition of additional phase modulation may serve to wash out these resonances. In the calculation of Fig. 6 (b) 1D-SSD using FM of four color cycles is supplemented by the superposition of FM of a single color cycle (i.e. FM at $1 / 4$ the frequency of the first modulator) and equal bandwidth (four times the modulation depth of the first modulator). It should be emphasized that this calculation is for 1D-SSD using two FM's (one modulation frequency four times the other) in series before dispersion. One sees that the resonances are strongly modified by the additional FM. Of course, in the limit of the superposition of many FM's one simply reproduces the effect of RPM. However, by proper choice of a small number of multiple FM's one may be able to obtain effective smoothing at low spatial frequency while minimizing the coherence resonances.
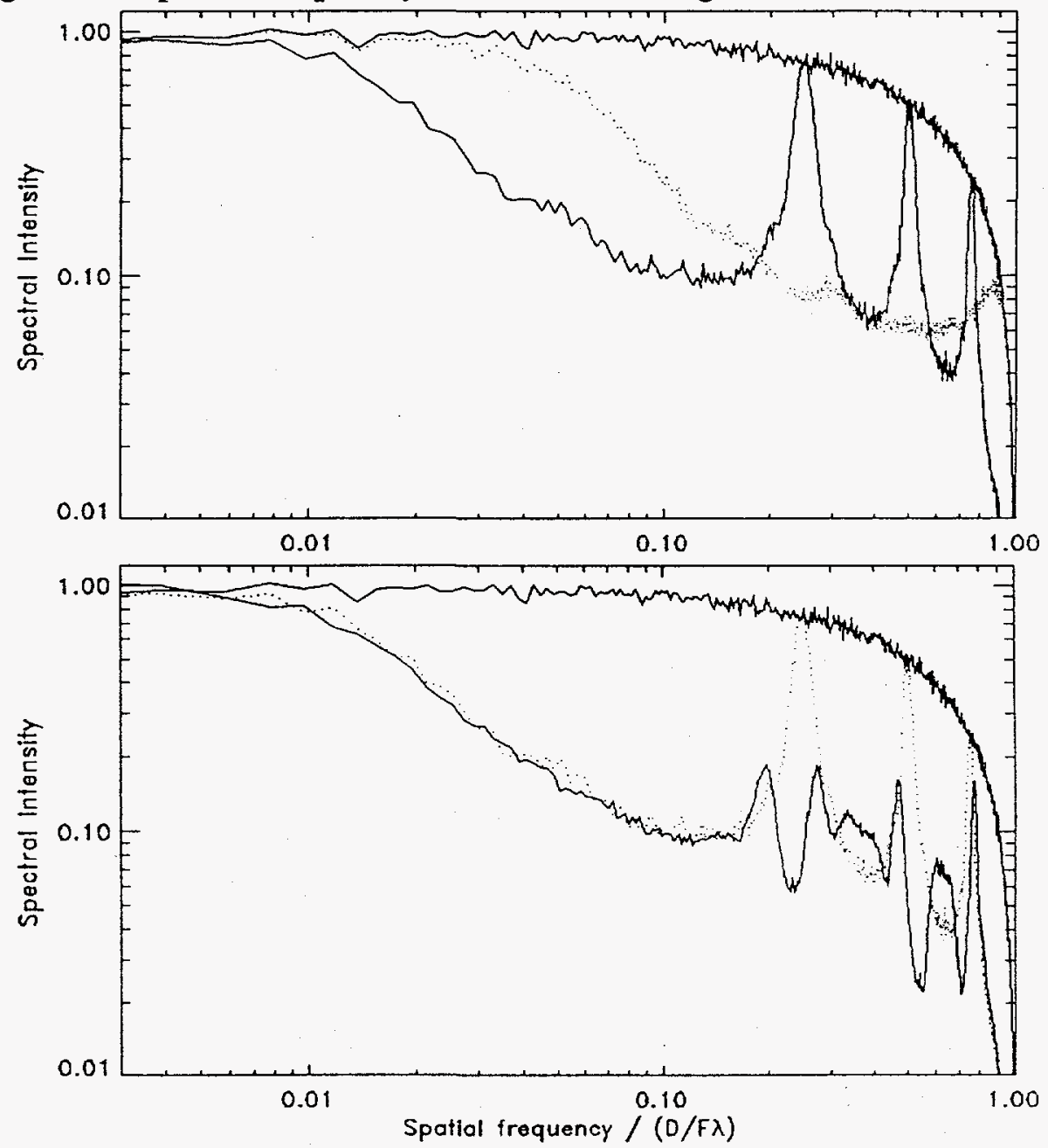

Figure 6: (a) Spatial spectra of 1D SSD with FM of a single color cycle (dotted curve) and four color cycles (lower solid curve) of equal bandwidth and divergence after an integration time equivalent to 4 coherence times. (b) Spatial spectra of 1D SSD with FM of four color cycles (dotted curve, as in (a)), and then modified with the superposition of an additional FM (still 1D-SSD) of a single color cycle of equal bandwidth (lower solid curve). The upper solid curve in both plots is the static speckle spectrum. 
Finally, concerning the practical implementation of RPM, one must insure that the phase modulation is sufficient to insure that the axial "coherent spike" is suppressed. This coherence effect (unrelated to that of the color cycles) is absent so long as the RPM has phase RMS $\phi_{0}$ greater than $\sim \pi \mathrm{rad}^{2}$ The implications of this requirement become apparent if one first notes that the total bandwidth FWHM of the RPM light is given by

$$
\Delta v_{\text {total }}=\Delta v_{\text {phase }} \cdot \phi_{0},
$$

where $\Delta v_{\text {phase }}$ is the bandwidth FWHM of the phase. After dispersion, the angular divergence FWHM of the beam is then given by

$$
\begin{aligned}
\Delta \theta & =T_{\text {skew }} \cdot \Delta v_{\text {total }} \cdot(\lambda / D) \\
& =N_{c c} \cdot \phi_{0} \cdot(\lambda / D)
\end{aligned}
$$

If we now assume $\Delta \theta$ is limited by a maximum $\Delta \theta_{\max }$, and take $\phi_{0}=\pi$ as the minimum phase depth, then one finds that $\Delta \theta_{\max } \geq N_{c c} \cdot \pi \cdot(\lambda / D)$ or

$$
N_{c c} \leq \Delta \theta_{\max } / \pi \cdot(\lambda / D) \text {. }
$$

Thus, e.g., in the NIF geometry $\lambda / D=1 \mu \mathrm{rad}$, and taking $\Delta \theta_{\max } \sim 50 \mu \mathrm{rad}$, implies that $N_{c c} \leq 16$, which, as shown in Figs. 4 and 5, is adequate to get optimal smoothing at low spatial frequencies.

\section{Conclusions}

It has been shown that SSD using FM of a single color cycle is ineffective (compared with ISI, for example) at smoothing of low spatial frequencies. Since the smoothing performance at low spatial frequencies may be of significance for both the direct and indirect drive approaches, it is important to examine methods which improve the smoothing rate at these low spatial frequencies. It has been shown that the smoothing rate at low spatial frequencies is proportional to the (mean) number of color cycles of the phase modulation across the beam. However, the use of a single sinusoidal FM of many color cycles leads to coherent resonances in the spatial spectrum. These resonances can be eliminated by either using RPM or perhaps with multiple FM's. The smoothing performance of SSD using RPM is equivalent to that obtained with an ISI-type method of an equal beam divergence and bandwidth.

\section{References}

1. J. E. Rothenberg, NIF-LLNL-95-388, July 21, 1995, see Eq. (4).

2. J. W. Goodman, "Statistical properties of laser speckle patterns", in Topics in Applied Physics, J. C. Dainty ed., vol. 9, pp. 63-74, Springer-Verlag, New York, 1984). 


\section{DISCLAMMER}

This report was prepared as an account of work sponsored by an agency of the United States Government. Neither the United States Government nor any agency thereof, nor any of their employees, makes any warranty, express or implied, or assumes any legal liability or responsibility for the accuracy, completeness, or usefulness of any information, apparatus, product, or process disclosed, or represents that its use would not infringe privately owned rights. Reference herein to any specific commercial product, process, or service by trade name, trademark, manufacturer, or otherwise does not necessarily constitute or imply its endorsement, recommendation, or favoring by the United States Government or any agency thereof. The views and opinions of authors expressed herein do not necessarily state or reflect those of the United States Government or any agency thereof. 

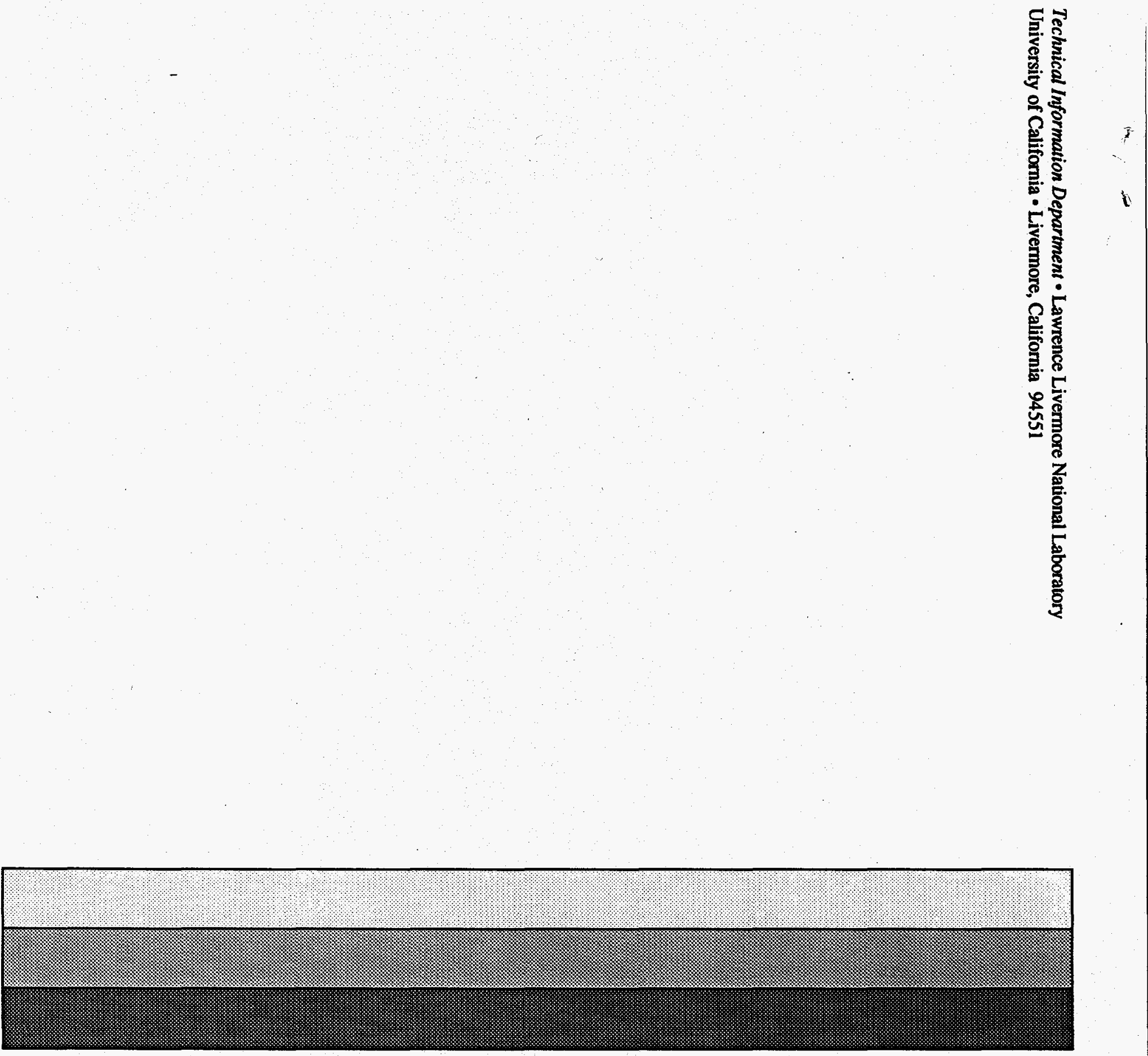

$63 \begin{aligned} & \text { Recycled } \\ & \text { Recyclable }\end{aligned}$ 